\title{
Methodology for Intraoperative Laser Doppler Vibrometry Measurements of Ossicular Chain Reconstruction
}

\author{
Jacek Sokołowski $\cdot$ Magdalena Lachowska $\cdot$ Robert Bartoszewicz $\cdot$ Kazimierz Niemczyk \\ Department of Otolaryngology, Medical University of Warsaw, Warsaw, Poland
}

Objectives. Despite the increasing number of research concerning the applications of the Laser Doppler Vibrometry (LDV) in medicine, its usefulness is still under discussion. The aim of this study is to present a methodology developed in our Department for the LDV intraoperative assessment of ossicular chain reconstruction.

Methods. Ten patients who underwent "second look" tympanoplasty were involved in the study. The measurements of the acoustic conductivity of the middle ear were performed using the LDV system. Tone bursts with carrier frequencies of $500,1,000,2,000$, and $4,000 \mathrm{~Hz}$ set in motion the ossicular chain. The study was divided into four experiments that examined the intra- and interindividual reproducibility, the utility of the posterior tympanotomy, the impact of changes in the laser beam angle, and the influence of reflective tape presence on measurements.

Results. There were no statistically significant differences between the two measurements performed in the same patient. However, interindividual differences were significant. In all cases, posterior tympanotomy proved to be useful for LDV measurements of the ossicular prosthesis vibrations. In most cases, changing the laser beam angle decreased signal amplitude about $1.5 \%$ (not significant change). The reflective tape was necessary to achieve adequate reflection of the laser beam.

Conclusion. LDV showed to be a valuable noncontact intraoperative tool for measurements of the middle ear conductive system mobility with a very good intraindividual repeatability. Neither a small change in the angle of the laser beam nor performing the measurements through posterior tympanotomy showed a significant influence on the results. Reflective tape was necessary to obtain good quality responses in LDV measurements.

Keywords. Otitis Media; Hearing Loss, Conductive; Ear, Middle; Ossicular Prosthesis; Tympanoplasty

\section{INTRODUCTION}

Despite the increasing number of research concerning the applications of the Laser Doppler Vibrometry (LDV) in medicine, its usefulness is still under discussion. LDV has been described as a very sensitive and noncontact method. This feature is used for

- Received April 7, 2015

Revised April 27, 2015

Accepted April 28, 2015

- Corresponding author: Magdalena Lachowska

Department of Otolaryngology, Medical University of Warsaw, ul. Banacha

1a, 02-097 Warsaw, Poland

Tel: +48-22-5992125, Fax: +48-22-5992123

E-mail: mlachowska@wum.edu.pl measurements that assess movements of the middle ear components. The frequencies used range from $100 \mathrm{~Hz}$ to $25,000 \mathrm{~Hz}$. This technique might be a supplementary method to impedance audiometry in middle ear testing [1,2]. In addition, it allows obtaining information about the mobility of the ossicular chain. In the literature, this is confirmed by the results of several other experiments [3-5]. However, the methodology of those measurements still presents some questions without answers. Repeatability of measurements performed during surgery, the impact of changes in the laser beam angle on results, and the necessity to use the reflective element have been still problems to be solved.

The aim of this study was to present a methodology developed in our Department for the LDV intraoperative assessment

Copyright (C) 2016 by Korean Society of Otorhinolaryngology-Head and Neck Surgery.

This is an open-access article distributed under the terms of the Creative Commons Attribution Non-Commercial License (http://creativecommons.org/licenses/by-nc/4.0)

which permits unrestricted non-commercial use, distribution, and reproduction in any medium, provided the original work is properly cited. 
of ossicular chain reconstruction.

\section{MATERIALS AND METHODS}

Four experiments were conducted. In experiment \#1, \#2, and \#3, 10 patients were involved. Experiment \#4 was performed in the laboratory. All of the enrolled patients underwent "secondlook" (second stage) tympanoplasty 12 months after the first stage surgery due to otitis media with cholesteatoma. The average age of the subjects was 67.6 years (women, 69.3 years; men, 65.0 years; range, 51 to 84 years). Antromastoidectomy with posterior wide tympanotomy were performed during the "second-look" procedure. Facial nerve, chorda tympani, tympanic membrane were preserved in all cases. The surgical technique using "long columella" ossiculoplasty was performed. In this technique, ossicular prosthesis was inserted between the tympanic membrane and the stape's footplate. Measurements of ossicular chain movements were performed using the LDV System (Polytec GmbH, Waldbronn, Germany). In this method, the Doppler effect for the light wave is used to make noncontact vibration measurements. In this technique the Doppler effect (the change in frequency of a wave reflected from the surface of interest that moves relatively to wave's source, also called Doppler shift) in a laser beam was used to measure the displacement velocity of reflecting surface. The laser beam from LDV was directed at the surface of interest. In case of this study the surface of interest was ossicle prosthesis (Fig. 1). The vibration amplitude and frequency were extracted from the Doppler shift of the reflected laser beam frequency, and the velocity of movement was then calculated by the computer system.

The LDV System was controlled by LMS TestXpress software (LMS International, Leuven, Belgium). Acoustic waves with carrier frequencies of 500, 1,000, 2,000, and 4,000 $\mathrm{Hz}$ were gen-

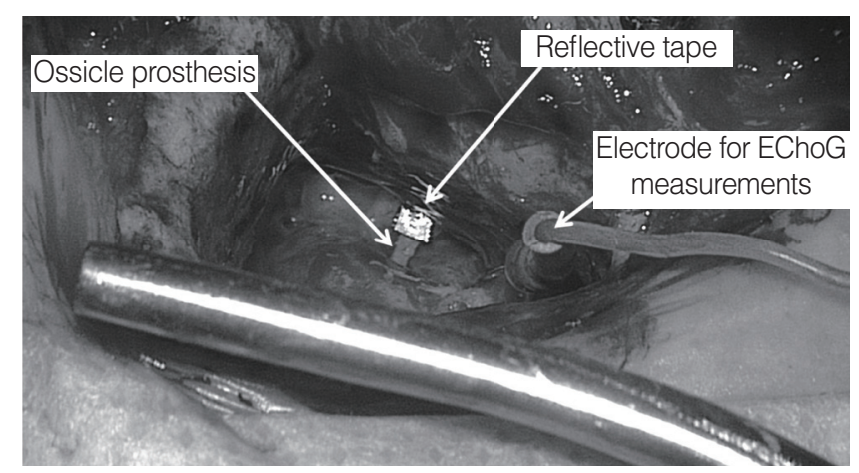

Fig. 1. Reflective foil attached to the "long columella" prosthesis just before Laser Doppler Vibrometry measurements started. View through the posterior tympanotomy. EChoG, electrocochleaography; this electrode was used in addition for acoustical hearing monitoring during surgery (auditory evoked potentials measurements) that are not presented in this study. erated by IHS System (Intelligent Hearing Systems, Miami, FL, USA) and applied to the ears using insert earphones ER2 (insert ear phones ER2, Etymotic Research Inc., Elk Grove Village, IL, USA), one frequency at a time. The stimuli were applied with the intensity of 90-dB sound pressure level (SPL) as tone bursts lasting $8 \mathrm{~ms}$ each and rate of 25 repetitions/sec. The stimuli set the ossicular chain in motion and its vibration was measured using LDV system.

This study is a part of a prospective project that was approved by the local Ethics Committee Review Board at the Medical University, where the project has been conducted. The project conforms with The Code of Ethics of the World Medical Association (Declaration of Helsinki).

\section{Experiment \#1}

Reproducibility of the obtained measurements within the same patient was assessed in this part of the study. In this experiment, ten patients were enrolled. All patients underwent "secondlook" surgical procedure with long columella ossiculoplasty. A sterile piece of an aluminum reflective tape (with the diameter of $1 \mathrm{~mm} \times 1 \mathrm{~mm}$ ) was attached to the "long columella" prosthesis (Fig. 1). A sinusoidal signal with the carrier frequency of 500, 1,000, 2,000, and 4,000 $\mathrm{Hz}$ (one at a time) and intensity level of 90-dB SPL was applied to the ear via ER2 earphones setting ossicular chain into motion. The measurements were performed twice, each lasting 5 seconds.

The vibrations of the measured structure were recorded and recognized by comparing the characteristics of the signal and noise. The frequency of the outgoing laser beam was compared with reflected laser beam modulated by the velocity (vibration) of the reflecting surface (the "long columella" prosthesis with the reflective tape). The response depends on the amplitude of the reflected laser beam returning to the velocity decoder. The sound-to-noise ratio (SNR) was calculated for each measurement. Spectral components at stimulus carrier frequencies $(500,1,000$, 2,000 , and $4,000 \mathrm{~Hz}$ ) were compared to the component magnitudes at the frequencies of $\pm 250, \pm 255, \pm 260 \mathrm{~Hz}$ from the response. The response was assessed as present if the SNR was higher than the average noise level plus 3 standard deviations.

The laser head was positioned $1.5 \mathrm{~m}$ from the surgical field. Laser beam was directed to the reflective tape placed on the prosthesis. Anatomic limits for the laser beam during the measurements were the edges of the tympanotomy, the external auditory canal, and sigmoid sinus. These anatomic limits allowed for maximum change in laser beam angle of about 10-12 degrees. A percentage ratio of the reflected laser beam to the outgoing one reached $80 \%-100 \%$, and was an indicator of correct conditions of measurements.

\section{Experiment\#2}

Typical intraoperative approach to the middle ear ("canal wall up" tympanoplasty) and interindividual reproducibility of the 

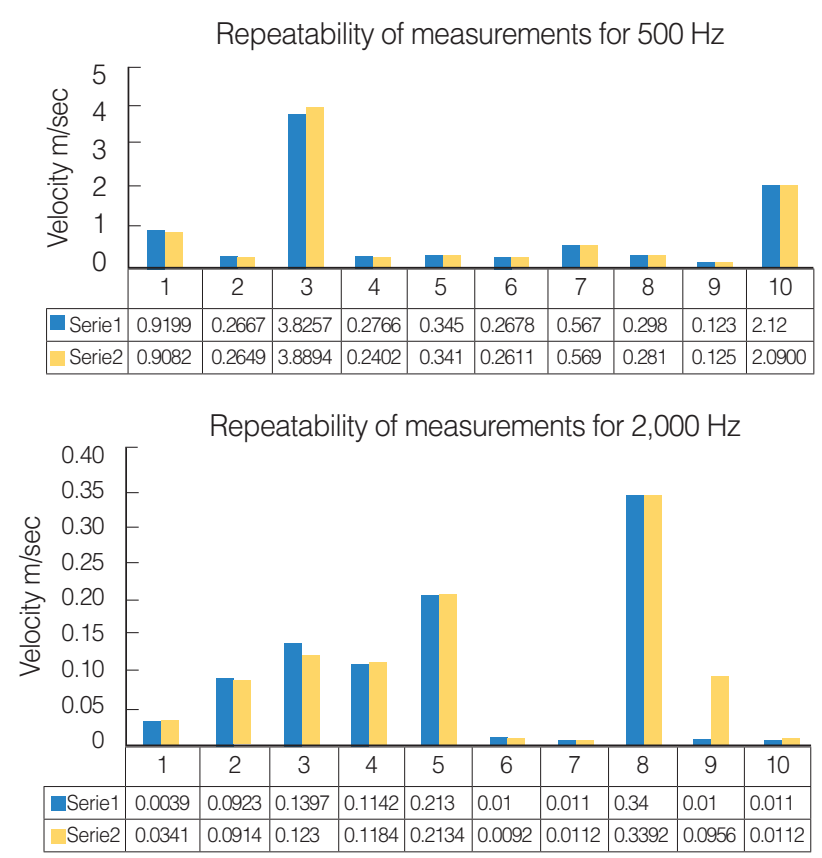

Repeatability of measurements for $1,000 \mathrm{~Hz}$

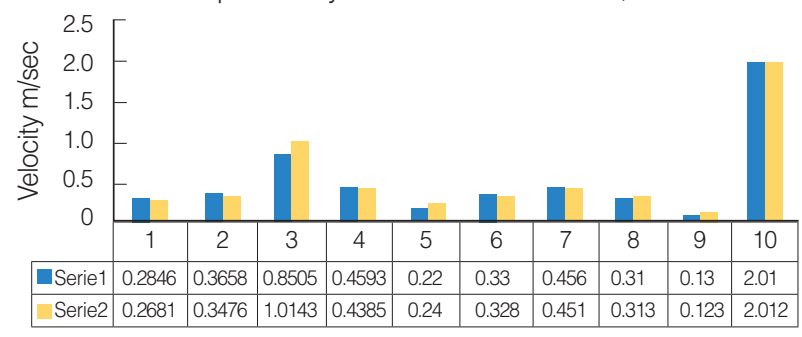

Repeatability of measurements for $4,000 \mathrm{~Hz}$

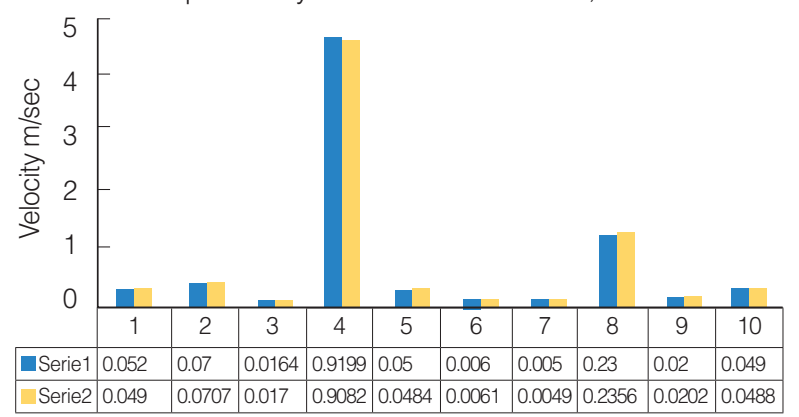

Fig. 2. The average velocity measured for each carrier frequency in each case separately $(n=10)$. The horizontal axis shows the patient number, and the vertical axis represents the averaged measured velocity.

method (10 cases) were examined in this experiment. Similar to the experiment $\# 1$, ossicular chain was stimulated using the same acoustic stimuli and methodology. Sterile piece of an aluminum reflective tape was attached to the ossicular prosthesis. Single measurement time was 5 seconds. During that time the average of 85.13 sweeps (median, 77 sweeps) was collected.

\section{Experiment \#3}

The impact of a piece of the reflective aluminum tape presence on reproducibility of the measurements was evaluated in this experiment. The experiment was performed in 10 patients during "second-look" surgery. In each case, the tympanic membrane was fully healed from the first surgery and without any perforation. Antromastoidectomy with wide posterior tympanotomy was performed. The ossicular chain was reconstructed using autologous long columella prosthesis. The same stimulation and signal acquisition method was used as in the experiment \#1. The laser beam was directed onto the surface of the prosthesis, next round window membrane, and next onto promontory with and without the reflective element attached to it. The area of each of the measured points presented different reflection properties. Ossicular chain prosthesis made from autologous bone was not covered by mucosa. However, the round window membrane (the tympanic cavity surface) and the promontory were covered by the mucous membrane. The velocity of tested point movements with and without reflective tape on it was measured in this experiment.

\section{Experiment \#4}

The impact of changing the outgoing laser beam angle directed at the tested point on measurements was assessed in this experiment. The sterile piece of reflective aluminum tape $(3 \mathrm{~mm} \times 3$ $\mathrm{mm})$ was applied on the MDR-E818LP loudspeaker's membrane (Sony, Tokyo, Japan). Pure tones with the carrier frequencies of 500,1,000, 2,000, and 4,000 Hz and at 90-dB SPL intensity level were generated and send to the speaker. The measurements were performed using the same laser's head (OFV534, Polytec $\mathrm{GmbH}$ ). The laser's head was attached to a tripod that measured the angle of the laser beam. The angle of the laser beam was set to $10^{\circ}$ and then changed to $30^{\circ}$ during the experiment. Measurements were performed for each angle.

\section{Statistical analysis}

Statistical analysis of collected data was performed using Statistica ver. 9 (StatSoft Inc., St.Tulsa, OK, USA). The data were tested for normality, parametric and nonparametric criteria. To analyze the data the following tests were used: Mann-Whitney $U$ test and Wilcoxon signed rank test. A $P$-value of $<0.05$ was considered statistically significant.

\section{RESULTS}

\section{Experiment \#1}

The mobility of the long columella after having performed the wide tympanotomy was measured in this experiment. Two sets of recordings were collected in each patient to compare intrain- 


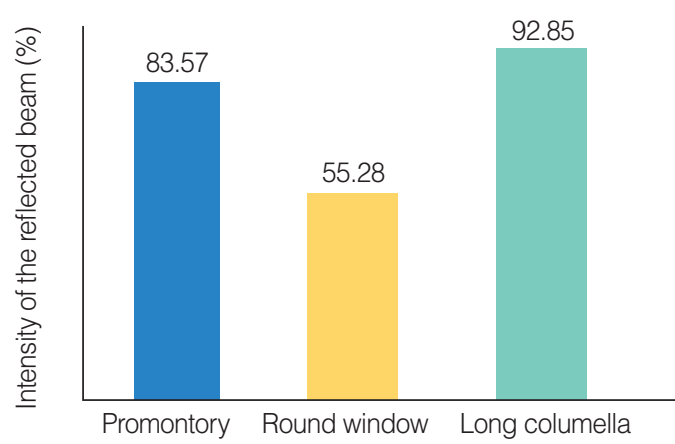

Fig. 3. The intensity of the reflected beam (\%) in regards to the surface of interest with the attached reflective tape. The highest reflectance was obtained from the ossicular prosthesis and the lowest from the round window.

dividual repeatability of measurements. The following average number of sweeps was collected: 105 sweeps at $500 \mathrm{~Hz}, 77.75$ sweeps at $1,000 \mathrm{~Hz}, 80.25$ sweeps at $2,000 \mathrm{~Hz}$, and 75 sweeps at $4,000 \mathrm{~Hz}$.

There were no statistically significant differences between the two measurements performed in each patient $(P>0.05)$ for each carrier stimulus frequency. The average velocity for each measured carrier frequency is shown in the Fig. 2.

Average SNR for $1,000 \mathrm{~Hz}$ amounted 108.78 (range, 28 to 198). For other carrier frequencies the SNRs were as follow: for $500 \mathrm{~Hz}, 180.16$ (range, 106 to 327); for 2,000 Hz, 18.30 (range 12 to 29 ); and for $4,000 \mathrm{~Hz}, 4$ (range, 3 to 5.4). SNR values were significantly higher than background noise for all measurements $(P<0.001)$.

\section{Experiment \#2}

Approximately 100 sweeps were recorded during the each 5 seconds measurement to evaluate interindividual reproducibility of the method. The average calculated prosthesis velocity was $1.31 \mathrm{~m} / \mathrm{sec}( \pm 1.7)$ for the stimulus with the carrier frequency of $500 \mathrm{~Hz}$. For the other carrier frequencies $(1,000,2,000$, and $4,000 \mathrm{~Hz})$ the speed was $0.53 \mathrm{~m} / \mathrm{sec}( \pm 0.30), 0.105 \mathrm{~m} / \mathrm{sec}( \pm$ $0.059)$, and $0.046 \mathrm{~m} / \mathrm{sec}( \pm 0.027)$, respectively. There was a large interindividual spread of results $(P<0.05)$ (Fig. 2).

\section{Experiment \#3}

It was not possible to obtain the reflectance from the mucous membrane of the tympanic cavity. Such measurements were however possible when reflective tape was attached to the surface of interest. The intensity of the reflected beam in those cases ranged from $80 \%$ to $100 \%$. The differences in the reflected beam intensities depended on the place of measurement (surface of interest with the attached reflective tape). The highest reflectance was obtained from ossicular prosthesis and amounted to $92.85 \%( \pm 11.39 \%)$, and from promontory $83.57 \%$ (standard deviation, $14.46 \%$ ). The lowest average values of reflection

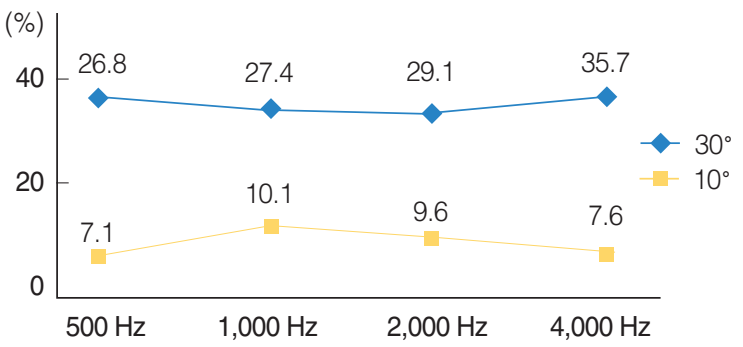

Fig. 4. The percentage change of the reflected laser beam with regard to the $30^{\circ}$ (blue color) and $10^{\circ}$ (yellow color) change in the angle of the outgoing laser beam. As shown, the $10^{\circ}$ angle change results in less change in reflected laser beam than $30^{\circ}$

intensities were recorded from the round window and amounted to $55.28 \%$ ( $\pm 11.60 \%)$ (Fig. 3 ).

\section{Experiment \#4}

Changing the angle of the outgoing laser beam about $30^{\circ}$ resulted in decreased amplitude of the response. Average reduction of the amplitude was about $29.74 \%$. Changing the angle to $10^{\circ}$ resulted in average of $8.61 \%$ decrease in amplitude (Fig. 4).

\section{DISCUSSION}

Main goals of middle ear surgery are removal of chronic inflamed tissues and improvement of hearing as final result. The pure tone audiometry (PTA) is usually very useful in assessing the hearing after the surgery. In PTA, closure of the air-bone gap shows the improved and proper function of the ossicular prosthesis. Unfortunately, subjective hearing testing cannot be used during the surgery. For these purpose electrophysiological methods such as electrocochleography or auditory brain stem responses are of great importance and usefulness. The idea of intraoperative assessment of the ossicular chain reconstruction presents a challenge for researchers and clinicians [6-8]. Over the decades many methods have been developed to assist the surgeon in measurements of the ossicular chain mobility. Initially, a contact method using the properties of a human hair was evaluated. This method was useful only in the experimental conditions. In the 80's and 90's noncontacting video and piezoelectric methods were introduced for measuring the mobility of the ossicular chain [9]. LDV is another method for assessing the ossicular chain reconstruction and offers safe intraoperative measurements.

In our study in experiment $\# 1$, reproducibility of the measurements within the same patient was assessed. As we expected, there were no significant differences between the measurements $(P>0.05)$. Responses measured at $1,000-\mathrm{Hz}$ carrier fre- 
quency were more difficult to detect. In those cases, larger but still not significant differences between the measurements were observed. In our study, we used the audiological criteria for SNR. The response was recognized as present when the recorded signal amplitude was higher than the average noise level plus three standard deviations.

In experiment \#2, large spread of results was obtained. In their experiment, Huber et al. [5] showed lower stapes mobility by about $5-10 \mathrm{~dB}$ (below $2 \mathrm{kHz}$ ) measured in vivo. Hydration state of the temporal bone specimen affected the LDV measurements $[10,11]$. However, Rosowski et al. [12] in their study did not find any statistical significance in the mobility of the tympanic membrane measured in laboratory and in the operating theater conditions. Ruggero and Temchin [13] found that differences between the measurements in vivo and in vitro resulted from postmortem decrease in muscle tension. Another explanation might be methodological differences in the measurements. In our study, after our experience in experimental measurements described in our previous study [14], middle ear acoustic conductivity was examined intraoperatively during "canal wall up" tympanoplasty. This surgical approach allows for the assessment of the ossicular chain mobility in its almost natural conditions. In Chien et al. [11]'s research, the measurements of an angle between the laser beam and the piston-like direction of stapes motion was estimated intraoperatively and was found to vary between $40^{\circ}$ and $60^{\circ}$. Such differences in the measurements might be important factors adding more variations to the measurements. In our study, the maximum possible change in the angle of the laser beam not affecting the measurements (proved in experiment \#4) was less than $10^{\circ}$ and was dependent on the anatomic limitations. In our study (in experiments \#1, \#2, and \#3), the angle change was within $3^{\circ}$ to $5^{\circ}$. The other authors suggested that very small angle changes (about $1.5 \%$ ) can be totally neglected. It must be, however, kept in mind that the measurement procedure we used differed from the methodology used in those other studies [15-17], therefore it would be difficult to compare the results from our study and those from other studies.

In our study, a piece of the reflective tape was attached to the ossicular prosthesis, promontory, and the round window. A percentage ratio of the reflected laser beam to the outgoing one reached $80 \%-100 \%$ when reflective tape was attached to the surface of interest. It was considered an indicator of correct conditions of measurement with good SNR. This stands in opposition to other authors presenting intraoperative results. They achieved a satisfactory reflection from the mucous membrane surrounding the stapes head without any reflective material. Huber et al. [18] showed that no reflective tape was required for the measurements of the tympanic membrane displacement. Chien et al. [19] showed that the reflective tape should not be used due to time required for its application/removal and the aseptic issues. In our study, we did not obtain sufficient reflection of the laser beam without the reflective tape. Thus sterile reflective tape was used during the measurements. Its application and removal took about 1 minute total.

In conclusion, LDV showed to be a valuable noncontact intraoperative tool for measurements of the middle ear conductive system mobility with a very good intraindividual repeatability. Neither a small change in the angle of the laser beam nor performing the measurements through posterior tympanotomy showed a significant influence on the results. Reflective tape was necessary to obtain good quality responses in LDV measurements.

\section{CONFLICT OF INTEREST}

No potential conflict of interest relevant to this article was reported.

\section{ACKNOWLEDGMENTS}

The study was supported by the Committee for Scientific Research grant (No. N 5180734 33).

\section{REFERENCES}

1. Whittemore KR Jr, Merchant SN, Poon BB, Rosowski JJ. A normative study of tympanic membrane motion in humans using a laser Doppler vibrometer (LDV). Hear Res. 2004 Jan;187(1-2):85-104.

2. Dai C, Gan RZ. Change of middle ear transfer function in otitis media with effusion model of guinea pigs. Hear Res. 2008 Sep;243(1-2):78-86.

3. Rosowski JJ, Merchant SN, Ravicz ME. Middle ear mechanics of type IV and type $\mathrm{V}$ tympanoplasty: I. model analysis and predictions. Am J Otol. 1995 Sep;16(5):555-64.

4. Zenner HP, Freitag HG, Linti C, Steinhardt U, Rodriguez Jorge J, Preyer S, et al. Acoustomechanical properties of open TTP titanium middle ear prostheses. Hear Res. 2004 Jun;192(1-2):36-46.

5. Huber A, Linder T, Ferrazzini M, Schmid S, Dillier N, Stoeckli S, et al. Intraoperative assessment of stapes movement. Ann Otol Rhinol Laryngol. 2001 Jan;110(1):31-5.

6. Huttenbrink KB. Mechanics of the ear ossicle chain in static pressures. I. The normal middle ear. Laryngol Rhinol Otol (Stuttg). 1988 Feb;67(2):45-52.

7. Merchant SN, Ravicz ME, Puria S, Voss SE, Whittemore KR Jr, Peake WT, et al. Analysis of middle ear mechanics and application to diseased and reconstructed ears. Am J Otol. 1997 Mar;18(2):139-54.

8. Niemczyk K, Sokolowski J, Bartoszewicz R, Morawski K, Bruzgielewicz A, Rygalska B. The methodology of measurements of ossicular chain movability during tympanoplasty using Laser Doppler vibrometry. Otolaryngol Pol. 2012 Mar-Apr;66(2):126-31.

9. Heiland KE, Goode RL, Asai M, Huber AM.A human temporal bone study of stapes footplate movement. Am J Otol. 1999 Jan;20(1):81-6.

10. Kirikae I. The structure and function of the middle ear. Tokyo: University of Tokyo Press; 1960.

11. Chien W, Ravicz ME, Merchant SN, Rosowski JJ.The effect of methodological differences in the measurement of stapes motion in live and cadaver ears. Audiol Neurootol. 2006 Mar;11(3):183-97.

12. Rosowski JJ, Mehta RP, Merchant SN. Diagnostic utility of laserDoppler vibrometry in conductive hearing loss with normal tympanic membrane. Otol Neurotol. 2003 Mar;24(2):165-75. 
13. Ruggero MA,Temchin AN. Middle-ear transmission in humans: wideband, not frequency-tuned? Acoust Res Lett Online. 2003 Mar;4:53-8.

14. Sokołowski J, Niemczyk K, Bartoszewicz R, Morawski K, Bruzgielewicz A. Method of ossicular chain valuation. Experimental measurement and clinical application. Otolaryngol Pol. 2009 Sep-Oct;63(5):432-6.

15. Goode RL, Ball G, Nishihara S, Nakamura K. Laser Doppler vibrometer (LDV): a new clinical tool for the otologist. Am J Otol. 1996 Nov;17(6):813-22.

16. Asai M, Heiland KE, Huber AM, Goode RL. Evaluation of a cement incus replacement prosthesis in a temporal bone model. Acta Oto- laryngol. 1999;119(5):573-6

17. Asai M, Huber AM, Goode RL.Analysis of the best site on the stapes footplate for ossicular chain reconstruction. Acta Otolaryngol. 1999;119(3):356-61.

18. Huber AM, Schwab C, LinderT, Stoeckli SJ, Ferrazzini M, Dillier N, et al. Evaluation of eardrum laser doppler interferometry as a diagnostic tool. Laryngoscope. 2001 Mar;111(3):501-7.

19. Chien W, Rosowski JJ, Ravicz ME, Rauch SD, Smullen J, Merchant SN. Measurements of stapes velocity in live human ears. Hear Res. 2009 Mar;249(1-2):54-61. 